\title{
Comparing cultivar means in multilocation trials when the covariance structure is not circular
}

\author{
HANS-PETER PIEPHO \\ University of Kassel, Steinstrasse 19, 37213 Witzenhausen, Germany
}

\begin{abstract}
Cultivar trials conducted at different locations are frequently evaluated by analysis of variance (ANOVA) techniques. One of the assumptions underlying such analyses is that residuals be independently distributed with common variance. Often, however, this assumption is violated. This contribution explores ways to analyse data when the usual assumptions regarding the variance-covariance structure do not hold. An example is given to demonstrate the proposed procedure.
\end{abstract}

Keywords: adjusted degrees of freedom, analysis of variance, circularity, genotype-environment interaction, paired $t$-test, stability variance.

\section{Introduction}

Multilocation trials are an important tool for evaluating the performance of crop cultivars. When analysing yield data from such trials, breeders are often interested in comparing the cultivar means across locations (Bradley et al., 1988). This is usually carried out by a two-way analysis of variance (ANOVA) followed by some multiple comparison procedure, e.g. the least significant difference test (LSD) (Johnson et al., 1992). Usually a mixed linear model with fixed genotypic effects and random environmental and interaction effects is assumed. For the ANOVA to be valid it is necessary that interactions and errors be independently distributed with homogeneous variances. Stated in slightly different terms, it is required that the vector of yields of the different genotypes in a specific environment follows a multivariate normal distribution with a variancecovariance matrix of the following form: (i) all diagonal elements are equal, i.e. all genotypes are equally variable across environments; (ii) all off-diagonal elements are equal, i.e. all pairs of genotypes are equally correlated. There is reason to believe, however, that this assumption is seldom met in practice.

The assumption regarding variances and covariances may have to be relaxed on two grounds (Calinski et al., 1987a,b). (i) The variance is not necessarily constant across genotypes. Heterogeneity of variances is often seen as an indication of stability differ- ences among genotypes, and such differences are often observed in practice. (ii) It is often found that some pairs of genotypes respond more similarly to varying environmental conditions than do others. For this reason, it is more realistic to make no restrictive assumptions regarding the covariances.

In the following a procedure will be discussed for dealing with these two departures from the assumption.

\section{Theory and method}

The usual model for genotype-environment data can be written

$y_{i j}=\mu+g_{i}+e_{j}+v_{i j}$

where $y_{i j}$ is the yield of the $i$ th genotype $(i=1, \ldots$, $K)$ in the $j$ th environment $(j=1, \ldots, N)$ and $\mu, g_{i}, e_{j}$, $v_{i j}$ are, respectively, the grand mean, the effect of the $i$ th genotype, the effect of the $j$ th environment and a residual corresponding to $y_{i j}$ comprising both experimental error and genotype-environment interaction. We will make the common assumption that environments constitute a random sample from some specified target region. This implies that both $e_{j}$ and $v_{i j}$ are random.

The usual assumption is that random effects are normally distributed with zero mean and common variance, i.e. $e_{j}$ has variance $\sigma_{e}^{2}$ and $v_{i j}$ has variance $\sigma_{v}^{2}$. This assumption is often referred to as compound symmetry (Winer et al., 1991, p. 243). 
Given compound symmetry, data may be analysed by usual ANOVA (see Table 1). Under the null hypothesis of no differences among cultivars across locations, the $F$-statistic for genotypes $\left(F_{\mathrm{G}}=\mathrm{MSG} /\right.$ MSR) is then distributed as central $F$ with $K-1$ and $(N-1)(K-1)$ degrees of freedom (d.f.).

It has been stated in the previous section that it may be more appropriate to assume an unrestricted covariance structure given by $\operatorname{Cov}\left(y_{i j}, y_{i^{\prime} j}\right)=\sigma_{i i^{\prime}}$. It is noted, however, that the usual ANOVA is appropriate under a more general condition than that of compound symmetry. This condition is referred to as circularity. The covariance structure is said to be circular if the quantity $\sigma_{i i}+\sigma_{i^{\prime} i^{\prime}}-2 \sigma_{i i^{\prime}}$ is constant for each pair $i, i^{\prime}\left(i<i^{\prime}\right)$ (Winer et al., 1991, p. 242). Note that this quantity is the variance of the difference between the observations of genotypes $i$ and $i^{\prime}$ in the same environment, i.e. $\operatorname{Var}\left(y_{i j}-y_{i^{\prime}}\right)$.

Given an unstructured variance-covariance matrix the linear model may be written as

$y_{i j}=\mu_{i}+\varepsilon_{i j}$,

where $\mu_{i}=\mu+g_{i}$ and $\varepsilon_{i j}$ is a random term corresponding to $y_{i j}$ with $\sigma_{i i^{\prime}}=\operatorname{Cov}\left(y_{i j}, y_{i^{\prime} j}\right)=\operatorname{Cov}\left(\varepsilon_{i j}, \varepsilon_{i^{\prime}}\right)$. It is also mentioned here that the model in eqn (2) may be restated as follows: the random vector $\mathbf{y}_{\mathbf{j}}=\left(y_{1 j}, y_{2 j}, \ldots, y_{K_{j}}\right)^{\prime}$ follows a multivariate normal distribution with means vector $\boldsymbol{\mu}=\left(\mu_{1}, \mu_{2}, \ldots, \mu_{K}\right)^{\prime}$ and unstructured variance-covariance matrix $\Sigma=\left\{\sigma_{i i^{\prime}}\right\}$.

In the case where the circularity condition is violated the distribution of $F_{\mathrm{G}}=\mathrm{MSG} / \mathrm{MSR}$ may be approximated by that of $F$ with $\varepsilon(K-1)$ and $\varepsilon(N-1)$ $(K-1)$ d.f. (Box, 1954), where $\varepsilon$ is a correction factor measuring the degree of departure from circularity. It can be shown that $\varepsilon$ lies between $(K-1)^{-1}$ and 1 . Thus, d.f. must be reduced. If departures from circularity are ignored, the real level of significance will exceed the nominal level established by the experimenter.

$\varepsilon$ is usually not known and has to be estimated from the data. One estimator has been suggested by Geisser \& Greenhouse (1958):

$\hat{\varepsilon}=\frac{K^{2}\left(\bar{s}_{i i}-s . .\right)^{2}}{(K-1)\left(\Sigma_{i} \Sigma_{i} s_{i i}^{2}-2 K \Sigma_{i} s_{i}^{2}+K^{2} s_{.}^{2} .\right)}$

where $s_{i i^{\prime}}=$ sample covariance of the $i$ th and $i^{\prime}$ th genotypes, $\quad \bar{s}_{i i}=K^{-1} \Sigma_{i} s_{i i}, \quad s . .=K^{-2} \Sigma_{i} \Sigma_{i^{\prime}} s_{i i^{\prime}} \quad$ and $s_{i} .=K^{-1} \sum_{i} s_{i i^{\prime}}$.

This estimator tends to be biased when the true $\varepsilon$ is close to unity. A modified estimator was suggested by Huynh \& Feldt (1976) which is preferable when $\varepsilon$ is not much smaller than unity:

$$
\tilde{\varepsilon}=\frac{N(K-1) \hat{\varepsilon}-2}{(K-1)[N-1-(K-1) \hat{\varepsilon}]} .
$$

Provided that $N>K$ we may test for circularity by Mauchley's sphericity criterion $W=\operatorname{det}(\mathbf{Q}) /$ $[\operatorname{tr}(\mathbf{Q}) /(K-1)]^{K-1}$, where $\mathbf{Q}=\mathbf{L S} \mathbf{L}^{\prime}, \mathbf{S}=\left\{s_{i i^{\prime}}\right\}$, and $\mathbf{L}$ is a $(K-1) \times K$ matrix of $K-1$ orthogonal contrasts such that $\mathbf{L} \mathbf{L}^{\prime}=\mathbf{I}_{K-1}\left(\mathbf{I}_{K-1}\right.$ is the $K-1$ dimensional identity matrix). Details are described by Winer $e t$ al. (1991), who also give exact critical values of $W$ for various combinations of $N$ and $K$. Unfortunately, when $N \leq K$, the test is inoperative. One may then resort to an inspection of the empirical variances and covariances $s_{i i^{\prime}}$ or the estimates of $\varepsilon$. Estimates of $\varepsilon$ smaller than 0.8 point to serious departure from circularity.

It may be noted that as an alternative to the $F$-test with correction of d.f., one may perform Hotelling's multivariate $T^{2}$-test (Graybill, 1954; Calinski et al., 1987a,b; Winer et al., 1991, p. 278ff.) to detect cultivar differences. This test essentially transforms the hypothesis

$\mu_{1}=\mu_{2}=\ldots=\mu_{K}$,

where $\mu_{i}=\mu+\mathrm{g}_{i}(i=1, \ldots, K)$, into the equivalent hypothesis

$\boldsymbol{\theta}=\left[\begin{array}{c}\mu_{1}-\mu_{K} \\ \mu_{2}-\mu_{K} \\ \cdot \\ \cdot \\ \mu_{K-1}-\mu_{K}\end{array}\right]=\left[\begin{array}{c}0 \\ 0 \\ \cdot \\ \cdot \\ 0\end{array}\right]$

This test is applicable only when $N>K$, i.e. when there are more environments than genotypes. Thus, when $N \leq K$, one is forced to resort to the $F$-test with adjusted d.f..

When circularity is violated, the usual procedures for comparing means are also inappropriate. These procedures (e.g. LSD) employ the residual mean square as a common error term for all comparisons. Under departure from circularity, however, each comparison of means may have a different variance. By employing the residual error mean square of a joint ANOVA of all cultivars there is a danger that the statistical significance of some comparisons will be under- or over-estimated. The simplest way to circumvent this problem is to perform a paired $t$-test for each pair of genotypes. This is equivalent to 
carrying out a two-way ANOVA for each pair of genotypes separately. The advantage of this procedure is that an unbiased estimate of the error variance is obtained for each comparison. It should be noted, however, that the paired $t$-test entails a loss of d.f., since we only have $(N-1)$ d.f. compared to $(K-1)$ $(N-1)$ d.f. for the LSD test.

Both the LSD and paired $t$-tests control only the comparison-wise error rate. In order to control the family-wise error rate (FWE) it may be preferable to use the Tukey test instead of the LSD test. For other tests see Hochberg \& Tamhane (1987). A more general approach, which is also applicable to the multiple paired $t$-test, is to adjust the significance level by the Bonferroni procedure. In order to control the FWE at $\alpha$, the paired comparisons are performed at $\alpha / c$, where $c=K(K-1) / 2$.

It may be useful to realize that two models commonly used in stability analysis imply a departure from the circularity condition, namely the regression model (Finlay \& Wilkinson, 1963: see Appendix 1) and the stability variance model (Shukla, 1972). These two models are more restrictive than eqn (2). In fact, they may be regarded as special cases of eqn (2), just as the ordinal mixed model in eqn (1) with homogeneous variances.

Under the mixed model in eqn (1) with the common assumptions we have $\varepsilon_{i j}=e_{j}+v_{i j}$, $\sigma_{i i}=\sigma_{e}^{2}+\sigma_{v}^{2}$ and $\sigma_{i i^{\prime}}=\sigma_{e}^{2}$. The same specifications hold for Shukla's model, except that $\sigma_{i i}=\sigma_{e}^{2}+\sigma_{v i}^{2}$, where $\sigma_{v i}^{2}$ is the 'stability variance' of the $i$ th genotype. Also, the regression model (with random locations; see Shukla, 1972) is a special case of this

Table 1 ANOVA table for multilocation data

\begin{tabular}{lcc}
\hline Source & Degrees of freedom & MS \\
\hline Location & $N-1$ & MSL \\
Genotype & $K-1$ & MSG \\
Residual & $(N-1)(K-1)$ & MSR \\
\hline
\end{tabular}

general model with $\varepsilon_{i j}=\beta_{i} e_{j}+\delta_{i j}$, where $\beta_{j}$ is the regression coefficient of the $i$ th genotype and $\delta_{i j}$ is a random deviation from the regression. The usual ANOva $F$-test (Table 1 ) is not valid under Shukla's model or the regression model, because the circularity condition is violated (see Table 2).

It is noted that mean yield and stability are two different features of a variety, so mean comparisons are no substitute for stability analysis. The important point is that Shukla's model and the regression model are compatible with the assumption of an unstructured covariance matrix, since they may be regarded as special cases of eqn (2). Thus, with both the regression model and Shukla's model, the procedures suggested in this paper are valid, while the usual ANOVA is not.

\section{Example}

In order to demonstrate the procedures outlined in this paper, we employ a data set published by Graybill (1954), which comprises the yields of four wheat cultivars tested in each of 13 locations. The data are displayed in Table 3. A simple sas program for performing the $F$-test with adjusted d.f. is given in Appendix 2.

Mauchley's sphericity criterion was computed as $W=0.0828$, which is significant at the 1 per cent level. The 5 per cent and 1 per cent critical levels for $K=4$ and $N=13$ are, respectively, 0.1938 and 0.1218 (Nagarsenkar \& Pillai, 1973; see also Winer et al., 1991, pp. 1006-1007). Values of $W$ lower than the critical value are regarded as significant. Departure from circularity is also suggested from inspection of the variances and covariances shown in Table 4.

Since circularity was rejected by Mauchley's test, the usual ANOVA was not deemed appropriate, and d.f. were adjusted by the Geisser-Greenhouse $\varepsilon$ and the Huynh-Feldt $\varepsilon$. The results of the $F$-tests are shown in Table 5 . The usual $F$-test as well as the adjusted d.f. $F$-tests were significant, although the

Table 2 Covariance structures under different models for two-way data

\begin{tabular}{|c|c|c|c|c|}
\hline Model & $\sigma_{i i^{\prime}}$ & $\sigma_{i i}$ & $\operatorname{Var}\left(y_{i j}-y_{i^{\prime} j}\right)$ & Circularity? \\
\hline $\begin{array}{l}\text { Compound symmetry } \\
\text { Stability variance } \\
\text { Regression model }\end{array}$ & $\begin{array}{c}\sigma_{e}^{2} \\
\sigma_{e}^{2} \\
\beta_{i} \beta_{i^{\prime}} \sigma_{e}^{2}\end{array}$ & $\begin{array}{c}\sigma_{e}^{2}+\sigma_{v}^{2} \\
\sigma_{e}^{2}+\sigma_{v i}^{2} \\
\beta_{i}^{2} \sigma_{e}^{2}+\sigma_{\delta i}^{2}\end{array}$ & $\begin{array}{c}2 \sigma_{v}^{2} \\
\sigma_{v i}^{2}+\sigma_{t i^{\prime}}^{2} \\
\sigma_{\delta i}^{2}+\sigma_{\delta i^{\prime}}^{2}+\left(\beta_{i}-\beta_{i^{\prime}}\right)^{2} \sigma_{e}^{2}\end{array}$ & $\begin{array}{l}\text { Yes } \\
\text { Usually not } \\
\text { Usually not }\end{array}$ \\
\hline
\end{tabular}


significance level of the usual $F$-test was rather higher than those of the other two tests. This reflects the fact that the usual $F$-test produces spurious significant results under departures from circularity. In comparison, the $F$-value of Hotelling's $T^{2}$-test was 7.59, with associated d.f. of 3 and 10 and a $P$-value of 0.0062 , which is highly significant.

Cultivar means were compared by the paired $t$-test. Each test was performed at $\alpha=0.05 / 6 \approx 0.0083$

Table 3 Wheat yield data of Graybill (1954)

\begin{tabular}{lrrrr}
\hline & \multicolumn{4}{c}{ Genotype } \\
\cline { 2 - 5 } Environment & 1 & 2 & 3 & 4 \\
\hline 1 & 43.60 & 24.05 & 19.47 & 19.41 \\
2 & 40.40 & 21.76 & 16.61 & 23.84 \\
3 & 18.08 & 14.19 & 16.69 & 16.08 \\
4 & 19.57 & 18.61 & 17.78 & 18.29 \\
5 & 45.20 & 29.33 & 20.19 & 30.08 \\
6 & 25.87 & 25.60 & 23.31 & 27.04 \\
7 & 55.20 & 38.77 & 21.15 & 39.95 \\
8 & 55.32 & 34.19 & 18.56 & 25.12 \\
9 & 19.79 & 21.65 & 23.31 & 22.45 \\
10 & 46.24 & 31.52 & 22.48 & 29.28 \\
11 & 14.88 & 15.68 & 19.79 & 22.56 \\
12 & 7.52 & 4.69 & 20.53 & 22.08 \\
13 & 41.17 & 32.59 & 29.25 & 43.95 \\
\hline
\end{tabular}

Table 4 Variance-covariance matrix for Graybill (1954) data

\begin{tabular}{lcrrr}
\hline & \multicolumn{4}{c}{ Genotype } \\
\cline { 2 - 5 } Genotype & \multicolumn{1}{c}{1} & \multicolumn{1}{c}{2} & \multicolumn{1}{c}{3} & 4 \\
\hline 1 & 262.81 & 137.91 & 6.07 & 74.72 \\
2 & & 88.43 & 11.83 & 54.61 \\
3 & & & 11.55 & 20.15 \\
4 & & & & 65.96 \\
\hline
\end{tabular}

(Bonferroni adjustment) to control the FWE at 5 per cent (Table 7). Results of an LSD test, with the same Bonferroni adjustment, are shown for comparison (Table 6). The grouping of means differed for the two procedures. According to the LSD test genotype 1 had a significantly higher yield than genotypes 2 and 3 . By contrast, the paired $t$-test declared the first genotype different only from genotype 2. Also, it detected a difference between genotypes 3 and 4, which was not found by the LSD test. This may be explained by the fact that the LSD test employs a common error term (the residual mean square of the ANOVA was 56.30; see Table 5). In contrast, a separate ANOVA for each pair of genotypes (equivalent to the paired $t$-test) yields a small residual mean square for some pairs and a larger mean square for others (Table 8). The comparison among genotypes 3 and 4 , for example, had a residual mean square of 18.60 , which is rather smaller than 56.30. The value of 18.60 is small enough for the paired $t$-test to declare genotypes 3 and 4 different (despite a loss of d.f.), whereas the LSD test does not find this difference.

\section{Discussion}

Yield data from multilocation trials are very commonly analysed by an ordinary two-way ANOVA without checking the validity of the circularity condition. This paper has demonstrated how d.f. may be adjusted in the case of departure from circularity. Although the adjusted d.f. $F$-test is widely used in sociological and psychological research (Huynh \& Feldt, 1976), few agricultural and biological scientists seem to be aware of this option.

Departure from circularity may result from heterogeneity of variances among genotypes, heterogeneity of covariances or both. In either case d.f. should be adjusted. For this procedure it is not necessary to decide on the type of noncircularity. However, such distinction may be necessary in a

Table 5 ANOva of Graybill (1954) data

\begin{tabular}{|c|c|c|c|c|c|c|c|}
\hline \multirow[b]{2}{*}{ Source } & \multirow[b]{2}{*}{ d.f. } & \multirow[b]{2}{*}{ SS } & \multirow[b]{2}{*}{ MS } & \multirow[b]{2}{*}{$F$} & \multicolumn{3}{|c|}{ Significance level } \\
\hline & & & & & Usual & $\mathrm{G}-\mathrm{G} \dagger$ & $\mathrm{H}-\mathrm{F} \ddagger$ \\
\hline Location & 12 & 3118.16 & 259.85 & & & & \\
\hline Genotype & 3 & 1106.57 & 368.85 & 6.55 & 0.0012 & 0.0158 & 0.0137 \\
\hline Residual & 36 & 2026.91 & 56.30 & & & & \\
\hline
\end{tabular}

$\lceil F$-test d.f. adjusted according to Geisser-Greenhouse; $\hat{\varepsilon}=0.4323$.

$\ddagger F$-test d.f. adjusted according to Huynh-Feldt; $\tilde{\varepsilon}=0.4628$.

(c) The Genetical Society of Great Britain, Heredity, 76, 198-203. 
Table 6 Mean comparisons among genotypes of Graybill (1954) data by LSD (with Bonferroni adjustment)

\begin{tabular}{ll}
\hline Genotype & Means \\
\hline 1 & $33.29^{\mathrm{a}}$ \\
2 & $24.05^{\mathrm{b}}$ \\
3 & $20.70^{\mathrm{b}}$ \\
4 & $26.16^{\mathrm{ab}}$ \\
\hline
\end{tabular}

Means followed by the same letter are not statistically different at $P=0.05 / 6 \approx 0.0083$ by the LSD test.

Table $7 P$-values of mean comparisons among genotypes of Graybill (1954) data by paired $t$-test

\begin{tabular}{lcccc}
\hline Genotype & 1 & 2 & 3 & 4 \\
\hline 1 & & 0.0024 & 0.0159 & 0.0789 \\
2 & & & 0.1923 & 0.2786 \\
3 & & & & 0.0072 \\
\hline
\end{tabular}

$P$-values $<P=0.0083$ are judged significant by the Bonferroni procedure.

Table 8 Error mean squares for ANOva based on pairs of genotypes from Graybill (1954) data

\begin{tabular}{lccc}
\hline & \multicolumn{3}{c}{ Genotype } \\
\cline { 2 - 4 } Genotype & 2 & 3 & 4 \\
\hline 1 & 37.70 & 131.11 & 89.66 \\
2 & & 38.16 & 22.59 \\
3 & & & 18.60 \\
\hline
\end{tabular}

related field, namely in stability analysis. In the case of heterogeneous covariances $\sigma_{i i}$, the appropriateness of Shukla's stability variance is doubtful, because it is based on a mixed model with $\sigma_{i i^{\prime}}=\sigma_{e}^{2}$, i.e. $\sigma_{i i^{\prime}}$ is assumed to be equal for all pairs of genotypes (see also Table 2). For the example given in the preceding section, homogeneity of $\sigma_{i i^{\prime}}$ was checked by the Wald test (Rao, 1973, pp. 418ff.). Homogeneity of $\sigma_{i i^{\prime}}$ was not rejected at the 5 per cent level. It is concluded that departure from circularity results from heterogeneity of variances and that Shukla's stability variance is an appropriate stability measure for the wheat data. Using Graybill's data, Snee (1982) detected heterogeneity among regression lines by the $F$-test of Mandel (1961), which might be seen as an indication of heterogeneous covariances (see Table 2). It should be stressed, however, that the $F$-test used by Snee is based on the assumption that under $\mathrm{H}_{0}$, interaction variances, and hence the variances $\sigma_{\mathrm{ii}}$, are homogeneous. The $F$-test for heterogeneity of regression lines is invalidated when interaction variances are heterogeneous. Moreover, as shown in Appendix 1, heterogeneity among regression coefficients may also occur when $\sigma_{i i^{\prime}}=\sigma_{e}^{2}$, namely when stability variances are heterogeneous. Thus, a significant test of heterogeneity of regression coefficients does not generally support the conclusion that covariances are heterogeneous.

\section{References}

BOX, G. E. P. 1954. Some theorems on quadratic forms applied in the study of analysis of variance problems. II. Effect of inequality of variance and of correlation between errors in the two-way classification. Ann. Math. Stat., 25, 484-498.

BRADLEY, J. P., KNITTLE, K. H. AND TROYER, A. F. 1988. Statistical methods in seed corn product selection. $J$. Prod. Agric., 1, 34-38.

CALINSKI, T, CZAJKA, S. AND KACZMAREK, Z. 1987a. A model for the analysis of a series of experiments repeated at several places over a period of years. I. Theory. Biul. Oceny Odmian, 17-18, 7-33.

CALINSKI, T, CZAJKA, S. AND KACZMAREK, Z. 1987b. A model for the analysis of a series of experiments repeated at several places over a period of years. II. Example. Biul. Oceny Odmian, 17-18, 35-71.

EBERHART, S. A. AND RUSSELL, w. A. 1966. Stability parameters for comparing varieties. Crop Sci., 6, 36--40.

FINLAY, K. W. AND WILKINSON, G. N. 1963. The analysis of adaption in a plant breeding programme. Aust. J. Agric. Res., 14, 742-754.

GEISSER, S. AND GREENHOUSE, s. w. 1958. An extension of Box's results on the use of the F-distribution in multivariate analysis. Ann. Math. Stat., 29, 885-891.

GRAYBILL, F. A. 1954. Variance heterogeneity in a randomized block design. Biometrics, 10, 516-520.

HOCHBERG, Y. AND TAMHANE, A. C. 1987. Multiple Comparison Procedures. John Wiley, New York.

HUYNH, H. AND FELDT, L. S. 1976. Estimation of the Box correction for degrees of freedom from sample data in randomized block and split plot designs. J. Educ. Stat., 1, 69-82.

IOHNSON, J. J., ALLDREDGE, J. R., ULLRICH, S. E. AND DANGI, o. 1992. Replacement of replications with additional locations for grain sorghum cultivar evaluation. Crop Sci., 32, 43-46.

MANDEL, J. 1961. Nonadditivity in two-way analysis of variance. J. Am. Stat. Ass., 56, 878-888.

NAGARSENKAR, B. N. AND PILLAI, K. C. S. 1973. The distribution of the sphericity test criterion. J. Multivariate Anal., 3, 226-235.

RAO, C. R. 1973. Linear Statistical Inference and its Application. John Wiley, New York.

(c) The Genetical Society of Great Britain, Heredity, 76, 198-203. 
SHUKLA, G. K. 1972. Some statistical aspects of partitioning genotype-environmental components of variability. Heredity, 29, 237-245.

SNEE, R. D. 1982. Nonadditivity in a two-way classification: is it interaction or nonhomogeneous variance? J. Am. Stat. Ass., 77, 515-519.

WINER, B. J., BROWN, D. R. AND MICHELIS, K. M. 1991. Statistical Principles in Experimental Design, 3rd edn. McGraw-Hill, New York.

\section{Appendix 1}

The regression coefficient of Finlay \& Wilkinson (1963) may be expressed as

$$
\beta_{i}=\frac{\operatorname{Cov}\left(y_{i j}, \bar{y}_{\cdot j}\right)}{\operatorname{Var}\left(\bar{y}_{\cdot j}\right)}
$$

With the general assumption that $\operatorname{Cov}\left(y_{i j}, y_{i^{\prime} j}\right)=\sigma_{i i^{\prime}}$, one finds

$$
\operatorname{Cov}\left(y_{i j}, \bar{y}_{j}\right)=K^{-1} \Sigma_{i^{\prime}} \sigma_{i i^{\prime}}
$$

and

$$
\operatorname{Var}\left(\bar{y}_{\cdot j}\right)=K^{-2} \Sigma_{i} \Sigma_{i^{\prime}} \sigma_{i i^{\prime}}
$$

It may be shown that generally $\Sigma_{i} \beta_{i}=K$, which is the usual constraint imposed on the regression model. Under compound symmetry, we have that $\beta_{i}=1$ for every $i$. Any departure from compound symmetry may cause heterogeneity among $\beta_{i} \mathrm{~s}$. Heterogeneity of $\beta_{i}$ s may also occur when all covariances are equal to the environmental variance, i.e. when interaction effects are uncorrelated, but the variances $\sigma_{i i}$ differ among genotypes. This implies heterogeneity among stability variances.

\section{Appendix 2}

The following is a simple sas program for performing the $F$-test for genotypes with adjusted d.f. as described by Geisser \& Greenhouse (1958) and Huynh \& Feldt (1976), Hotelling's $T^{2}$-test, and Machley's sphericity test.

DATA;

INPUT $\mathrm{X} 1 \quad \mathrm{X} 2 \quad \mathrm{X} 3 \quad \mathrm{X} 4 ; \quad{ }^{*} \mathrm{X} 1-\mathrm{X} 4=$ yields $\mathrm{OF}$ GENOTYPES 1 TO $4 * 1$

CARDS;

43.6024 .0519 .4719 .41

$l^{*}$ data for other 12 locations*

;

PROC GLM;

MODEL X1 $-\mathrm{X} 4=$ /NOUNI;

REPEATED GENOTYPE/PRINTE;

RUN; 